\title{
Desarrollo tecnológico de paneles multicapa a partir de residuos lignocelulósicos bioligados con micelio de hongos.
}

\section{Technological development of multilayer panels from lignocellulostic waste bioliged with fungi mycelium.}

Presentación: 11/10/2019

Doctorando:

\author{
Natalia Fernández \\ (CEVE-AVE-CONICET) \\ nfernandez@ceve.org.ar
}

\section{Director/es:}

\section{Dra. Rosana Gaggino, Dr. Jerónimo Kreiker, Dra. María Josefina Positieri}

\begin{abstract}
Resumen
Esta tesis forma parte de la línea investigativa que propone el desarrollo de componentes constructivos utilizando residuos, a partir de los desechos lignocelulósicos y el micelio de hongos para generar alternativas sustentables a productos utilizados actualmente en viviendas tales como paneles aislantes elaborados con poliestireno expandido o lana de vidrio, que hoy son fabricados y aglutinados con resinas sintéticas en base a derivados del petróleo. El micelio es el aparato vegetativo de los hongos cuya función es absorber de su entorno los nutrientes de los compuestos orgánicos que necesite para alimentarse y está constituido por filamentos pluricelulares denominados hifas.
\end{abstract}

Palabras claves: Componentes constructivos, Residuos lignocelulósicos, Micelio.

\begin{abstract}
This thesis is part of the research line that proposes the development of construction components whit waste, from lignocellulosic waste and fungal mycelium to generate sustainable alternatives to products currently used in housing such as insulating panels made with expanded polystyrene or glass wool, which today are manufactured and bonded with synthetic resins based on petroleum derivatives. The mycelium is the vegetative part of fungi whose function is absorbing from their environment the nutrients of the organic compounds they need for food and consists of multicellular filaments called hyphae.
\end{abstract}

Keywords: Constructioncomponents, Lignocellulosicwastes, mycelium.

\section{Introducción}

En la naturaleza existe una interacción continua entre factores biológicos y no biológicos que produce una circulación ininterrumpida de materia (carbono, agua, azufre, nitrógeno, fósforo, etc.) entre el medio inerte o inorgánico (biotopos) y las comunidades vivientes (biocenosis). En cada comunidad existen conjuntos de seres vivientes con necesidades complementarias, de forma que unos son productores, otros consumidores y unos terceros descomponedores de los restos que se generan. Debido a esta constante circulación cíclica de la materia en cada ecosistema (biotopo+biocenosis) los restos que se generan son reutilizados constantemente, por lo que en la naturaleza no existen residuos de ningún tipo. Esta realidad natural de producción sin residuos debe hacernos 
reflexionar sobre la baja eficiencia de nuestros sistemas productivos y servir como punto de referencia para una nueva concepción del reciclaje e incluso de una nueva producción sin residuos, como única alternativa para evitar la degradación y desaparición irreversible de nuestros recursos, y, en definitiva, de nuestra propia sociedad industrial [1].

Desde un principio la Biotecnología fue considerada como creadora de nuevas industrias con bajas demandas energéticas. Ello se debe a que el crecimiento de microorganismos representa una fuente de energía renovable que disminuye la dependencia con respecto a los escasos y costosos depósitos fósiles existentes, o bien de los productos químicos de ellos derivados [2].

Los hongos tienen la capacidad de secretar una serie de enzimas que degradan los desechos de las plantas y utilizan algunos de sus productos para su desarrollo. El género Pleurotus, el más versátil, presenta una amplia gama de especies comestibles, que se adaptan a diferentes condiciones ambientales. Esto permite el desarrollo del cultivo a menores costos, en diferentes regiones geográficas del mundo, ya que se pueden utilizar cepas que resulten adecuadas para las diversas temperaturas reinantes en las regiones, siempre que éstas sean más o menos constantes. Las especies de Pleurotus (conocidos como hongos en forma de ostra o gírgolas) tienen la característica de ser xilófagos, por lo cual se cultivan en diversos sustratos (aserrín de maderas no resinosas o paja de distintas malezas provenientes de cultivos) obtenidos de sobrantes de la industria agrícola y maderera; facilitándose una gran adaptabilidad a una economía regional y permitiendo mayor facilidad en el proceso de preparación del sustrato para la inoculación del hongo [3].

El micelio crece en materiales celulósicos que en general son residuos. "Estos hongos se alimentan degradando los complejos estructurales de los residuos vegetales (lignina, hemicelulosa y celulosa) mediante un sistema enzimático específico y transformándolos en moléculas simples, fáciles de absorber" [4]. Es por lo tanto, un recurso natural renovable. En cambio, las resinas poliméricas son de alto costo y son derivadas del petróleo, por lo cual consumen un recurso no renovable.

\section{Objetivos}

General:

Desarrollar tecnológicamente un material sustentable a partir de residuos ligno-celulósicos y micelio, para brindar soluciones constructivas de bajo costo que promuevan la descontaminación del ambiente.

Específicos:

- Determinar los residuos lignocelulósicos como compuesto predominante y la cepa de hongo mas adecuados para el crecimiento del micelio disponibles en el medio local para la producción de componentes constructivos.

- Evaluar las propiedades Físicas, Mecánicas, de Durabilidad, Térmicas, Fuego, etc.

- Identificar aplicaciones constructivas de los compuestos en base a micelio y residuos lignocelulósicos.

- Verificar que el material resultante esté despojado de emisiones contaminantes en su fabricación, tóxicos en su vida útil o que requieran complicados procesos de transformación para su reciclaje.

\section{Antecedentes}

Como antecedentes internacionales se puede mencionar a Biohm, empresa británica fundada por EhabSyed que hace investigaciones sobre el micelio como material aislante "vegano" como parte de su sistema Triagomy, el cual presenta un tipo de construcción sustentable que crea estructuras duraderas y robustas a través del concepto de economía circular [5]. 
Otro antecedente son los ladrillos patentados por Philip Ross, micólogo de la Universidad de Stanford que estudia las diversas posibilidades para el uso de los hongos. En sus estudios encontró que cuando el micelio de los hongos se seca es resistente al fuego, al agua y aíslan de la humedad, pudiendo utilizarse para formar un material de construcción fuerte, ligero y ecológico. A partir de sus estudios, Ross patentó ladrillos de hongos a partir de Ganoderma Lucidum y paja de trigo, que se pueden ensamblar para cualquier tipo de estructura [6].

Los Paneles estructurales aislantes (PEA) a partir de hongos y desechos agrícolas en la construcción de EcovativeDesign, compañía estadounidense fundada por Eben Bayer y Gavin McIntyre que comercializa un producto denominado Mushroom ${ }^{\circledR I n s u l a t i o n, ~ e l ~ c u a l ~ a c t u ́ a ~ c o m o ~ u n ~ a i s l a n t e ~ a c u ́ s t i c o ~ y ~ t e ́ r m i c o ~ e n ~ p a r e d e s ~ d e ~ e d i f i c i o s . ~ E l ~}$ producto consiste en hifas empaquetadas de un hongo que previamente se ha cultivado [7].

Esta compañía en conjunto con el estudio de arquitectura The Living, se interesaron en la patente desarrollada por el micólogo Ross y desarrollaron una torre temporal de ladrillos de hongos, llamada "Hy-fi" para el Museo de Arte Moderno de Nueva York en 2014. Los ladrillos utilizados en esta torre, y patentados por Ecovative, fueron una variación de la técnica utilizada por Ross, producto de un estudio y desarrollo sobre sus propiedades constructivas [8].

"Hy-fi" era una torre circular de 13 metros de alto construida con 10.000 ladrillos orgánicos de micelio proporcionados por Ecovative. La parte superior de la torre estaba formada por ladrillos reflectantes que hacían rebotar la luz hacia abajo y los ladrillos de micelio actuaban como un aislante térmico. Dado el carácter biodegradable de los materiales utilizados, tras acabar la exhibición fue desmontada y los ladrillos fueron procesados y usados para abonar jardines públicos. Un año antes, Ecovative ya había construido la MushroomTiny House, primera casa ecológica con las paredes aisladas mediante el uso de la tecnología sustentable MycoFoam, que sustituye las tradicionales espumas fabricadas a partir del petróleo. En el transcurso de unos días, el aislamiento con hongos creció in situ entre dos maderas. Durante el mes siguiente el hongo se secó, y dejó como resultado una pared hermética extremadamente fuerte. Al ser una materia orgánica, el micelio terminaría muriendo a lo largo del primer mes, formando entonces un sello hermético frente a temperatura, humedad y fuego.

En el campo del desarrollo de materiales sustentables, EcovativeDesign, ofrece en el mercado paneles aislantes y de terminación compuestos por micelio que se pueden lijar, cortar, fresar y pintar. Particularmente desarrollaron tres tipos de paneles, que los denominan MycoComposite 029/570/584, que se diferencian entre sí por sus componentes y por ende en sus propiedades materiales resultantes. Este producto, de $97 \mathrm{~cm} \mathrm{x} 97 \mathrm{~cm} \mathrm{x} 5 \mathrm{~cm}$, es el producto en base a micelio de mayores dimensiones en el mercado global.

En Argentina, ya existen materiales cultivados a partir de bacterias y micelio de hongos con aplicaciones en el campo del diseño industrial desarrollados por Silvio Tinello, cuyo postulado es "la biología es la nueva tecnología" [9], y también podemos mencionar la iniciativa de la diseñadora experimental Heidi Jalkh y los biólogos Leonardo Majul y la Dra. Julieta Mallerman en TaMaCo de la cheLA-Fundación. El Taller de Materiales y Construcción realiza workshops de Bio-Fabricación, buscando explorar y promover la creación y uso de materiales formados a partir del crecimiento de organismos vivos, particularmente, el micelio del hongo Ganoderma Lucidum. Este organismo tiene la capacidad natural de fusionar y transformar diferentes tipologías de sustratos orgánicos que permite la exploración de las posibilidades formales del material y la realización de diversas piezas por medio de herramientas de fabricación digital. El objetivo que se plantea el curso es la exploración de las posibilidades materiales del hongo, con el propósito de entender sobre la forma y la materia en la bio- fabricación [10].

Por último, algunos estudios realizados en la escuela de Ingeniería de Sydney, Australia, han encontrado que los compuestos de micelio son una alternativa económica, sostenible y térmicamente más segura que los materiales de construcción sintéticos derivados del petróleo y el gas natural. En particular, los compuestos de micelio tuvieron tasas de liberación de calor promedio y pico mucho más bajas y un tiempo estimado de evaporación más prolongado que los materiales de construcción sintéticos considerados. También liberaron significativamente menos humo y CO2. Las cáscaras de arroz produjeron cenizas de carbón y sílice significativas que mejoraron el rendimiento frente al fuego, pero los compuestos que contienen finos de vidrio exhibieron el mejor rendimiento frente al fuego debido a 
sus concentraciones de sílice significativamente más altas y bajo contenido de material combustible. En general, los compuestos de micelio fueron muy económicos y exhibieron parámetros de seguridad contra incendios mucho mejores que los materiales de construcción tradicionales probados. Su uso generalizado en la construcción civil permitiría una mejor seguridad contra incendios en los edificios [11].

\section{Metodología}

En esta etapa inicial del proyecto se ha seguido la metodología desarrollada por Philip Ross con la cual obtuvo los ladrillos patentados citados anteriormente. Para el proceso de fabricación del material propuesto se seleccionaron dos materiales: un sustrato residual con alto contenido de celulosa y semilla de avena inoculada con las esporas de dos especies fúngicas disponibles en el medio local, que actuarán como ligante. La relación entre semillas y sustrato que se utilizó, para garantizar un óptimo desarrollo del micelio, fue de aproximadamente 1:10.

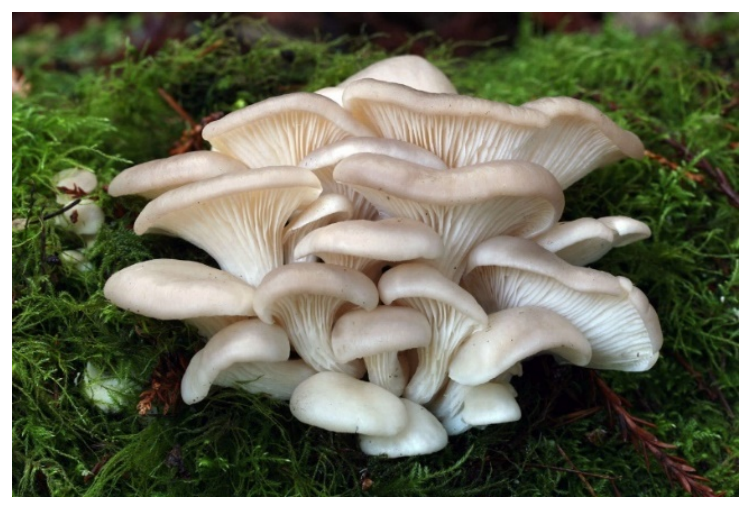

Fig. 1: Fruto del hongo Pleurotus Ostreatus o gírgola. Fuente: http://www.mykoweb.com

El método utilizado para el cultivo de este material fue el siguiente: en primer lugar, se aisló la cepa y se generó el inóculo. Estas primeras tareas se realizaron en institutos de biología con equipamiento especializado. Para iniciar esta investigación, se utilizaron las especies Pleurotus Ostreatus (Fig.1), y Ganoderma Lucidum (Fig.2) adquiridas en el IMBIV, considerando la posibilidad de ampliar el espectro de especies fúngicas en la medida en que se consigan esporas a través de instituciones que se desarrollen en el área de micología.

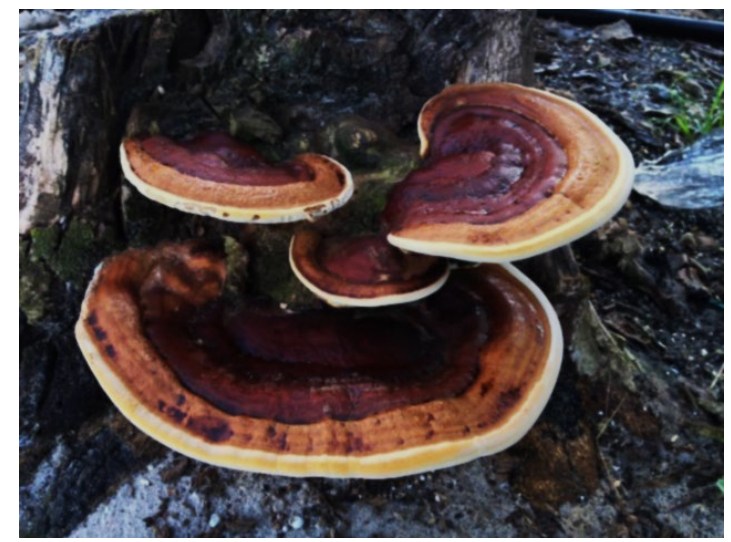

Fig. 2: Fruto del hongo Ganoderma Lucidum o Reishi. Fuente: https://mushroomobserver.org

Luego, se seleccionó el sustrato y se redujo la carga microbiana. Para esta primera etapa experimental se eligió el método de pasteurización por inmersión en agua a $80^{\circ}$ y $100^{\circ} \mathrm{C}$ durante 2 horas realizando distintas muestras para su posterior comparación. Una vez realizado este proceso se centrifugó el sustrato pasteurizado en una secadora de ropa con tambor de acero inoxidable previamente desinfectado con alcohol $70^{\circ}$. Se inoculó la semilla miceliada en el sustrato a una $\mathrm{T}^{\circ}$ ambiental no superior a los $30^{\circ}$ sobre una superficie lisa y desinfectada. Se colocó en moldes de madera igualmente desinfectados evitando que la mezcla quede en contacto con la madera mediante papel film, ya que el micelio podría adherirse a la superficie (Fig.3). 


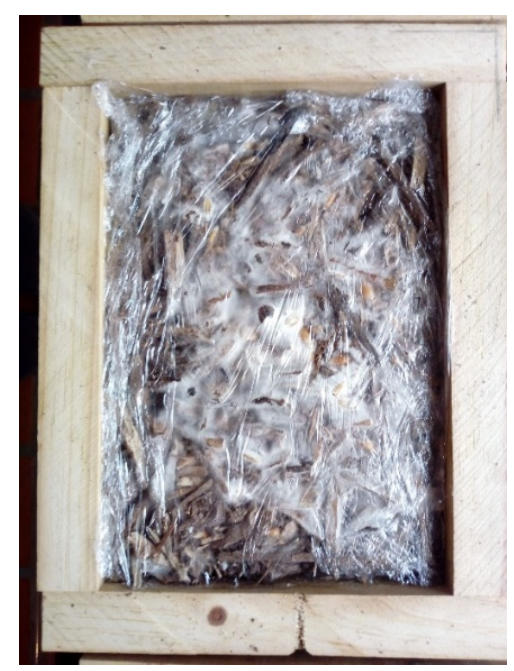

Fig 3: Molde con crecimiento micelial sobre sustrato de restos de poda de vid al día 5 de incubación. Fuente: Elaboración propia.

Se incubaron los moldes de las probetas en oscuridad durante 21 días en condiciones ambientales de $24^{\circ} \mathrm{C}$ y H${ }^{\circ}$ Rel. del 70\%. (Fig. 5 y 6). En algunos casos se dejó fructificar (Fig. 7 y 8). Finalmente, las muestras se desmoldaron y secaron en horno de esterilización a $70^{\circ}$ durante $24 \mathrm{~h}$ para detener el crecimiento del micelio.
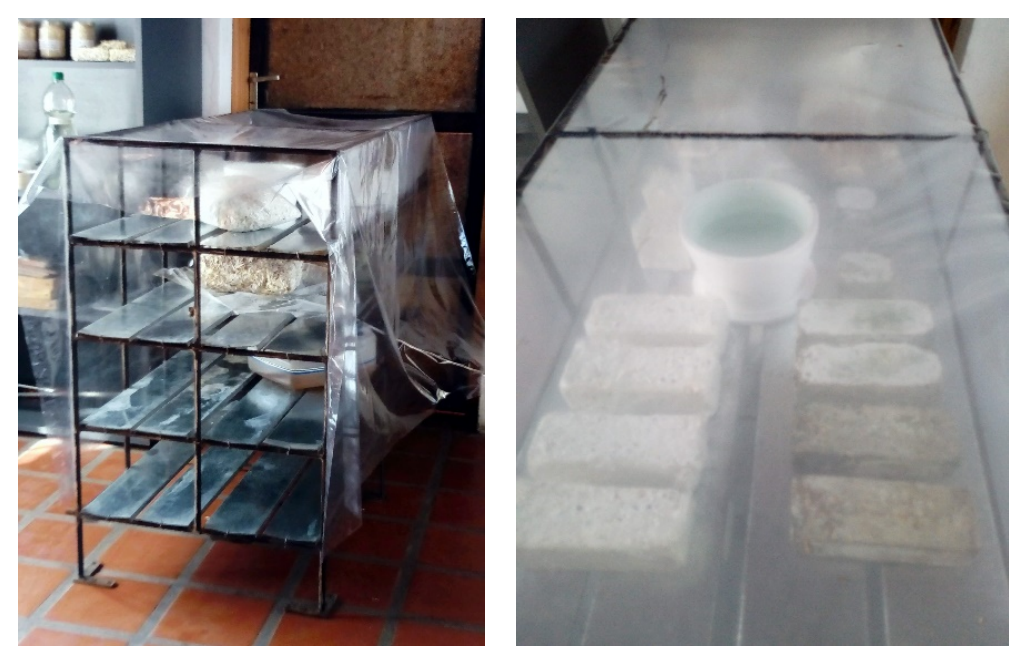

Fig 5 y 6: Cámara de incubación de probetas diseñada para mantener control de temperatura y humedad. Fuente: Elaboración propia.
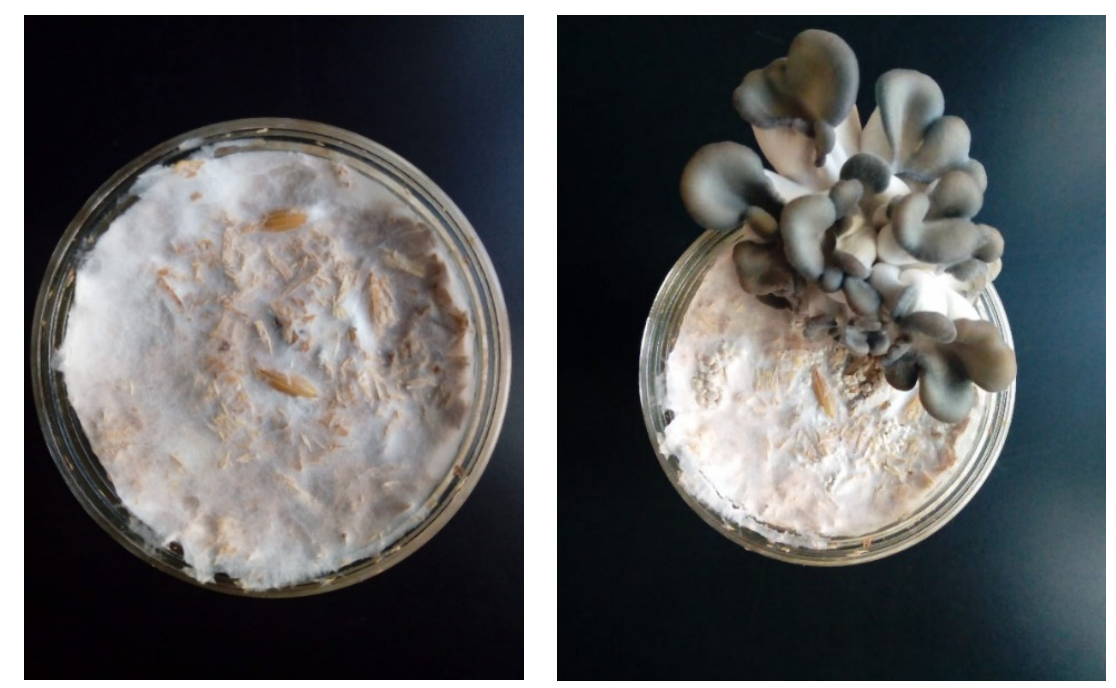

Fig 7 y 8: Micelio al día 21 de incubación y posterior fructificación de gírgola al día 25 cultivada en frasco de vidrio con sustrato de viruta de álamo. 
Para evaluar los mejores resultados, se propusieron combinaciones de diferentes sustratos utilizados como materia prima a partir de residuos lignocelulósicos triturados tales como:

\begin{tabular}{|l|l|}
\hline TIPOS DE SUSTRATO & PROCEDENCIA \\
\hline Hojas secas & Múltiples- Identificando especies \\
\hline Viruta de álamo & Industrial Maderera \\
\hline Virutas mixtas & Industrial Maderera- Carpinterías \\
\hline Bagazo de cerveza & Industrial Alimenticia \\
\hline Hollejo de Soja & Agrícola \\
\hline Cartón & Industrial Papelera \\
\hline Papel & Industrial Papelera \\
\hline Desmote de algodón & Agrícola \\
\hline Restos de yerba mate & Industrial alimenticia \\
\hline Borra de café & Comercial- Cafeterías \\
\hline Chips de poda urbana mixtas & Múltiples- Identificando especies \\
\hline Chips de poda de frutales & Agrícola \\
\hline Lana de oveja & Ganadero \\
\hline
\end{tabular}

\section{Resultados preliminares}

Se utilizaron para los experimentos dos cepas: Pleurotus Ostreatus (Gírgola) y Gano derma Lucidum (Reishi), obteniéndose una colonización del sustrato más homogéneo en la primera.

Se realizaron pruebas inoculando el hongo en sustratos de hojas secas de roble, viruta de álamo, virutas mixtas de palo amarillo, guatambú y cedro, cartón corrugado de doble onda, cartón de huevos, polypapel, borra de café, restos de yerba mate, bagazo de cebada, chips de poda de vid, lana de oveja, desmote de algodón, hollejo de soja, hojas de palma producto de poda urbana.

Los métodos de esterilización utilizados hasta el momento fueron pasteurización por inmersión en agua a $80^{\circ}$ y $100^{\circ}$ durante $2 \mathrm{~h}$, y el de tratamiento químico con solución alcalina del $0,7 \%$ del volumen total de sustrato. En el primer caso se observó contaminación en varias probetas, aunque en menor medida las de polypapel y cartón. Con el segundo método de esterilización, no se detectó contaminación del sustrato, pero si se observó que el micelio creció mejor en la parte superior de la muestra y no colonizó con el micelio la base.

Los casos de sustratos más desfavorables para el crecimiento del micelio han sido los siguientes:

- las hojas de roble, en donde las muestras se contaminaron con moho Trichoderma viride y Penicillyum.

-la lana de oveja con lignina, en la que el micelio no ha dado señales de crecimiento.

- el bagazo de cebada, que al ser un sustrato con mucho contenido de humedad se pudre con facilidad en el margen de $48 \mathrm{~h}$ luego de ser utilizado por la industria cervecera, con lo cual se debe pasteurizar rápidamente y es más propenso a contaminarse.

Los casos más favorables para el crecimiento del micelio han sido los siguientes:

-la pulpa de cartón corrugado de doble onda y el cartón de huevos utilizando como aditivo almidón de maíz en un $10 \%$ (Fig.9).

-el desmote de algodón en crudo, observando que podría mejorar en caso de triturarlo para reducir la granulometría.

- viruta de álamo y virutas variadas, principalmente utilizando la cepa Pleurotus Ostreatus.

- chips de poda de vid y de palma. 


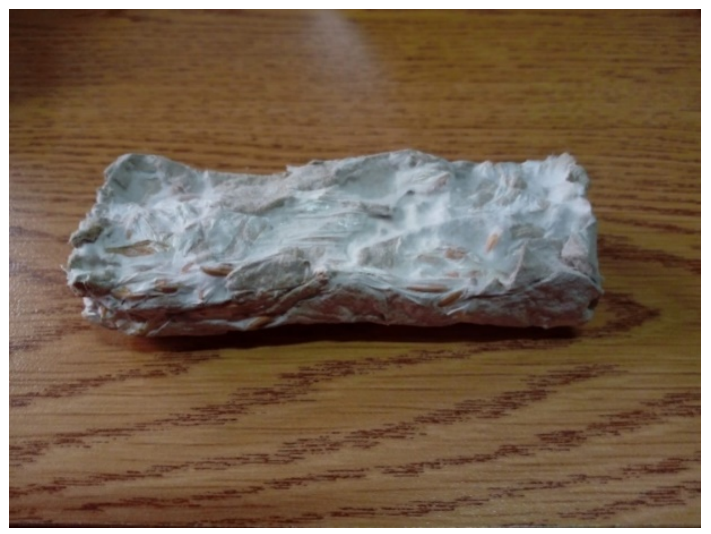

Fig 9: Material resultante de micelio con sustrato de cartón de huevos, una vez secado. Fuente: Elaboración propia.

En cuanto a la contaminación de las muestras, se deberá mejorar en etapas posteriores el método de esterilización, considerando reemplazar el método de pasteurización por el de tratamiento químico, esterilización con autoclave, o radiaciones. Otro factor a mejorar es la desinfección del ambiente en el cual es inoculada la semilla, en una cabina diseñada para tal fin.

Las probetas obtenidas con el procedimiento descripto tienen algunas propiedades técnicas ventajosas, según el análisis realizado en nuestro laboratorio experimental: liviandad, resistencia mecánica y baja conductividad térmica, por lo cual este material podría ser potencialmente usado en placas de aislación térmica. La siguiente etapa es realizar ensayos en el laboratorio del CINTEMAC de la UTN - FRC, para la determinación de las propiedades técnicas según normas.

\section{Referencias}

[1] DEL VAL, A. (2010). La Basura es un Tesoro. Suplemento especial Diario Los Andes. Argentina. pp.42-43.

[2] ALAN WISEMAN (1986). Principios de Biotecnología. Surrey, Reino Unido. Editorial Acribia. ISBN: 84-200-0572X. p.1

[3] ARIAS CARBAJAL, G. et al, (2005). Biotransformación de Residuos Lignocelulósicos con Hongos Pleurotus. Revista CENIC. Ciencias Biológicas, vol. 36, 2005 Centro Nacional de Investigaciones Científicas Ciudad de La Habana, Cuba. ISSN: 0253-5688

[4] GONZÁLEZ MATUTE, R. (2014). El Cultivo de Hongos Comestibles como forma de Reinserción Social. Bahía Blanca, Argentina. Boletín electrónico $\mathrm{N}^{\circ} 29$ CONICET. Disponible en: https://bahiablanca.conicet.gov.ar/boletin/boletin29/index56c7.html?option=com cont

[5] BIOHM (2016) Página del fabricante. Disponible en: https://www.biohm.co.uk/

[6] CARLOS, I. (2016). Hifas de hongos como material de construcción II- Revista Yesca 28: pp 19-22.

[7] ECOVATIVE DESIGNS (2016). Página del fabricante. Disponible en: https://ecovativedesign.com/

[8] BARICCI, R. (2016). Structural Analysis and Form-Finding of Mycelium-Based Monolithic Domes. Tesis doctoral. Scholarly Repository University of Miami.

[9] TINELLO (2018). Página del diseñador. Disponible en: https://www.silviotinello.com

[10] TaMaCo (2019). Página del estudio. Disponible en: https://tamaco.chela.org.ar/BIOFAB

[11] BATH et al, (2018).Biomass and Waste-derived Sustainable Mycelium Composite Construction Materials with Enhanced Fire Safety. Reseach Gate- Trabajo presentado en ECCM18 - 18th European Conference on Composite Materials 1 Athens, Greece, 24-28th June 2018. 